\title{
Step-Up and Step-Down Operators of a two-term Molecular Potential via Nikiforov-Uvarov Method
}

\author{
Altuğ Arda ${ }^{1, * *}$ and Ramazan Sever ${ }^{2}$, \\ ${ }^{1}$ Department of Physics Education, Hacettepe University, 06800, Ankara,Turkey \\ ${ }^{2}$ Department of Physics, Middle East Technical University, 06531, Ankara,Turkey
}

\begin{abstract}
The creation and annihilation operators of a two-term diatomic molecular potential are studied and it is observed that they satisfy the commutation relations of a $\mathrm{SU}(1,1)$ algebra. To study the Lie algebraic realization of the present potential, the normalized eigenfunctions and eigenvalues are computed by using the Nikiforov-Uvarov method.

Keywords: Ladder Operators, Nikiforov-Uvarov Method, diatomic potential, Schrödinger equation
\end{abstract}

PACS numbers: 03.65.-w, 03.65Ge

*E-mail: arda@hacettepe.edu.tr

†E-mail: sever@metu.edu.tr 


\section{INTRODUCTION}

The two-term diatomic molecular potential under consideration [1]

$$
V(r)=-V_{0} \frac{e^{-\beta r}}{1-q e^{-\beta r}}+V_{1} \frac{e^{-2 \beta r}}{\left(1-q e^{-\beta r}\right)^{2}},
$$

has been firstly proposed by Sun to study of some diatomic molecules. Jia and co-workers have studied the bound states of this potential for the Schrödinger (SE) equation in the view of supersymmetric approach [2] and in Ref. [3], the energy spectra of the potential is obtained in terms of Green's function for the same domain. Furthermore, Arda et. al. have studied the bound states and the corresponding normalized wave functions of this potential for the SE equation with any $\ell$-values for the cases where $q \geq 1$ and $q=0$. In addition, they have also given the analytical results for the Manning-Rosen potential, the Hulthén potential and the generalized Morse potential as special cases with their numerical results [4]. As a continuation, in the present work, we intend to search the creation and annihilation operators of the two-term molecular potential and find that the dynamical group satisfied by the ladder operators of the present potential. For this aim, we obtain firstly the normalized eigenfunctions of the potential by using the Nikiforov-Uvarov (NU) method [5] to present the ladder operators.

The group theoretical realizations within the quantum mechanics have been received great attentions in literature. The factorization method is used to get exact solutions of some solvable potentials with the ladder operators $[6,7]$. The perturbed ladder operator method is applied to quantum mechanical systems with the help of unperturbed eigenvalues and eigenfunctions $[6,8]$. Dong and co-workers have applied a 'different' factorization method to various types of molecules [9-16].

The organization of the present work is as follows. In Section 2, we search the normalized wave functions with their energy spectra of this potential by using NU-method which is

briefly given in Appendix A. In Section 3, we present the raising and lowering operators of the potential and show that the commutators of the ladder operators satisfy the Lie algebra of a $\mathrm{SU}(1,1)$ group. 


\section{NORMALIZED WAVE FUNCTIONS AND ENERGY SPECTRUM}

The radial Schrödinger equation is written [17]

$$
\frac{d^{2} R_{n \ell}(r)}{d r^{2}}+\left[\frac{2 \mu}{\hbar^{2}}\left[E_{n \ell}-V(r)\right]-\frac{\ell(\ell+1)}{r^{2}}\right] R_{n \ell}(r)=0
$$

where $\ell$ is the angular momentum quantum number, $m$ is the particle mass, $V(r)$ is the central potential and $E_{n \ell}$ is the non-relativistic energy.

Inserting Eq. (1) into Eq. (2) and using a new variable $x=q e^{-\beta r}(x \rightarrow q$ for $r \rightarrow 0$, $x \rightarrow 0$ for $r \rightarrow \infty)$ gives the following equation without the centrifugal term

$$
\frac{d^{2} R_{n}(x)}{d x^{2}}+\frac{1-x}{x(1-x)} \frac{d R_{n}(x)}{d x}+\left[-a_{1}^{2}-a_{2}^{2} x-a_{3}^{2} x^{2}\right] R_{n}(x)=0 .
$$

with the abbreviations

$$
\begin{aligned}
& a_{1}^{2}=-\frac{2 \mu E_{n}}{\beta^{2} \hbar^{2}}, \\
& a_{2}^{2}=\frac{2 \mu}{\beta^{2} \hbar^{2}}\left(2 E_{n}-\frac{V_{0}}{q}\right), \\
& a_{3}^{2}=\frac{2 \mu}{\beta^{2} \hbar^{2}}\left(\frac{V_{1}}{q^{2}}+\frac{V_{0}}{q}-E_{n}\right),
\end{aligned}
$$

where $V_{0}, V_{1}, \beta$ and $q$ are real parameters defined by $V_{1}=D_{0}\left(e^{\mu}-q\right), V_{0}=2 V_{1}, \beta=\mu / r_{0}$, where $D_{0}$ is the depth of the potential, $r_{0}$ is the equilibrium of the molecule and $q$ is the shape parameter.

Comparing Eq. (3) with Eq. (A1)we obtain

$$
\begin{aligned}
& \tilde{\tau}(x)=1-x, \\
& \sigma(x)=x-x^{2}, \\
& \tilde{\sigma}(x)=-a_{3}^{2} x^{2}-a_{2}^{2} x-a_{1}^{2},
\end{aligned}
$$

with $\sigma^{\prime \prime}(x)=-2$. Eq. (A7) gives

$$
\pi(x)=-\frac{x}{2} \mp \sqrt{\left(\frac{1}{4}+a_{3}^{2}-k\right) x^{2}+\left(a_{2}^{2}+k\right) x+a_{1}^{2}},
$$

We obtain the parameter $k$ is determined by setting the discriminant of the square root in Eq. (6)

$$
\begin{aligned}
& k_{1}=-a_{2}^{2}-2 a_{1}^{2}-a_{1} A, \\
& k_{2}=-a_{2}^{2}-2 a_{1}^{2}+a_{1} A ; \quad A=\sqrt{1+\frac{8 \mu V_{1}}{q^{2} \beta^{2} \hbar^{2}}} .
\end{aligned}
$$


From Eqs. (7a) and (7b) we get the followings for $\pi(x)$, respectively,

$$
\begin{aligned}
& \pi(x)=a_{1}-\left(a_{1}+\frac{1+A}{2}\right) x, \\
& \pi(x)=a_{1}-\left(a_{1}+\frac{1-A}{2}\right) x .
\end{aligned}
$$

The parameter $\lambda$ required for the method is obtained from Eq. (A8) with the help of Eq. $8(\mathrm{a})$

$$
\lambda=-a_{2}^{2}-2 a_{1}^{2}-\left(a_{1}+\frac{1}{2}\right)-A\left(a_{1}+\frac{1}{2}\right),
$$

and the other one $\lambda_{n}$ is written

$$
\lambda_{n}=n\left(2+2 a_{1}+A\right)+n(n-1)
$$

where used $\tau(x)=1+2 a_{1}-\left(2+2 a_{1}+A\right) x$ and $\tau^{\prime}(x)=-\left(2+2 a_{1}+A\right)$.

Setting $\lambda=\lambda_{n}$ and using the parameter values given in Eqs. (4a)-(4c) gives the following energy eigenvalue equation

$$
a_{1}+\frac{A}{2}=-\left(n+\frac{1}{2}\right) \mp a_{3},
$$

and the analytical expression for the energy spectra

$$
E_{n}=-\frac{\beta^{2} \hbar^{2}}{2 \mu}\left\{\frac{\left[2 n+1+\sqrt{1+\frac{8 \mu V_{1}}{q^{2} \beta^{2} \hbar^{2}}}\right]^{2}-\frac{8 \mu}{\beta^{2} \hbar^{2}}\left(\frac{V_{0}}{q}+\frac{V_{1}}{q^{2}}\right)}{4\left[2 n+1+\sqrt{1+\frac{8 \mu V_{1}}{q^{2} \beta^{2} \hbar^{2}}}\right]}\right\}^{2} .
$$

In order to find the wave functions, we first use Eq. (A6) to present the function $\rho(x)$

$$
\rho(x) \sim x^{2 a_{1}}(1-x)^{A}
$$

and inserting it into Eq. (A5) gives

$$
\varphi_{n}(x) \sim \frac{1}{x^{2 a_{1}}(1-x)^{A}} \frac{d^{n}}{d x^{n}}\left[x^{n+2 a_{1}}(1-x)^{n+A}\right],
$$

which means that the second part of the wave function in Eq. (A2) is written as the Jacobi polynomials [18]

$$
\varphi_{n}(x) \sim P_{n}^{\left(2 a_{1}, A\right)}(1-2 x)
$$

Eq. (A4) gives the first part of wave function as

$$
\psi(x) \sim x^{a_{1}}(1-x)^{(1+A) / 2}
$$


Finally, the total wave functions are given as

$$
R_{n}(x)=A_{n} x^{a_{1}}(1-x)^{(1+A) / 2} P_{n}^{\left(2 a_{1}, A\right)}(1-2 x) .
$$

where $A_{n}$ is a constant determined from the normalization condition. Choosing a new variable as $x=q y$, we write the normalization condition

$$
\int_{0}^{1}\left|A_{n}\right|^{2} q^{2 a_{1}} y^{2 a_{1}}(1-q y)^{1+A}\left[P_{n}^{\left(2 a_{1}, A\right)}(1-2 q y)\right]\left[P_{m}^{\left(2 a_{1}, A\right)}(1-2 q y)\right] d y=1
$$

Using the following representation of the Jacobi polynomials [18]

$$
P_{n}^{\left(\xi_{1}, \xi_{2}\right)}(z)=\frac{1}{2^{n}} \sum_{k=0}^{n}\left(\begin{array}{c}
a+n \\
k
\end{array}\right)\left(\begin{array}{c}
b+n \\
n-k
\end{array}\right)(1+z)^{k}(1-z)^{n-k}
$$

where binomial coefficient $\left(\begin{array}{l}n \\ r\end{array}\right)=\frac{n !}{r !(n-r) !}=\frac{\Gamma(n+1)}{\Gamma(r+1) \Gamma(n-r+1)}$. Hence, Eq. (18) becomes

$$
\left|A_{n}\right|^{2} q^{2 a_{1}+n+m-k-\ell+1}(-1)^{n+m-k-\ell}[g(n, k) \times g(m, \ell)] \int_{0}^{1} y^{2 a_{1}+n-k+m-\ell}(1-q y)^{1+A+k+\ell} d y=1,
$$

where $g(n, k)$ and $g(m, \ell)$ are two arbitrary functions of the parameters $a_{1}$ and $A$ and given by

$$
\begin{aligned}
& g(n, k)=\sum_{k=0}^{n}\left(\begin{array}{c}
n+2 a_{1} \\
k
\end{array}\right)\left(\begin{array}{c}
n+A \\
n-k
\end{array}\right) . \\
& g(m, \ell)=g(n, k)(n \rightarrow m ; k \rightarrow \ell),
\end{aligned}
$$

By using the following integral representation of hypergeometric type function ${ }_{2} F_{1}(a, b ; c ; z)$ [18]

$$
{ }_{2} F_{1}(a, b ; c ; z)=\frac{\Gamma(c)}{\Gamma(b) \Gamma(c-b)} \int_{0}^{1} t^{b-1}(1-t)^{c-b-1}(1-t z)^{-a} d t
$$

and by setting the variable $z \rightarrow q$ and taking $a=1+A+k+\ell, b=2 a_{1}+n+m-k-\ell+1$, $c=1+b$, Eq. (20) gives the normalization constant as

$$
A_{n}=\sqrt{\frac{q^{-2 a_{1}-n-m+k+\ell-1}(-1)^{k+\ell-n-m} \Gamma\left(2 a_{1}+n+m-k-\ell+2\right)}{g(n, k) g(m, \ell) \Gamma\left(2 a_{1}+n+m-k-\ell+1\right){ }_{2} F_{1}(a, b ; c ; q)}} .
$$




\section{CREATION AND ANNIHILATION OPERATORS}

We want to obtain the following eigenvalue equations for creation, $\hat{\Pi}_{+}$, and annihilation, $\hat{\Pi}_{-}$, operators

$$
\hat{\Pi}_{\mp}\left|n>=\pi_{\mp}\right| n>
$$

where the ket $\mid n>$ corresponds to the eigenfunctions $R_{n}(x)$. Firstly, we study the effect of the differential operator $\frac{d}{d x}$ on the eigenfunctions

$$
\frac{d}{d x}\left|n>=\left(\frac{a_{1}}{x}-\frac{1+A}{2(1-x)}\right)\right| n>+A_{n} x^{a_{1}}(1-x)^{(1+A) / 2} \frac{d}{d x} P_{n}^{\left(2 a_{1}, A\right)}(1-2 x),
$$

With the help of the following relation [18]

$$
\frac{d}{d x}\left[P_{n}^{\left(\eta_{1}, \eta_{2}\right)}(x)\right]=\frac{1}{2}\left(n+\eta_{1}+\eta_{2}+1\right) P_{n-1}^{\left(\eta_{1}+1, \eta_{2}+1\right)}(x)
$$

we obtain the first derivative in the last term of Eq. (25) as

$$
\frac{d}{d x}\left[P_{n}^{\left(2 a_{1}, A\right)}(1-2 x)\right]=-\left(n+2 a_{1}+A+1\right) P_{n-1}^{\left(2 a_{1}+1, A+1\right)}(x)
$$

Using the recursion relations for the Jacobi polynomials [18]

$$
\begin{aligned}
& P_{n}^{\left(\eta_{1}, \eta_{2}\right)}(x)=\frac{\eta_{1}+\eta_{2}+n+1}{\eta_{1}+n+1} P_{n}^{\left(\eta_{1}+1, \eta_{2}\right)}(x)-\frac{\eta_{2}+n}{\eta_{1}+n+1} P_{n}^{\left(\eta_{1}+1, \eta_{2}-1\right)}(x) \\
& P_{n}^{\left(\eta_{1}+1, \eta_{2}\right)}(x)=\frac{2}{2 n+\eta_{1}+\eta_{2}+2} \frac{1}{1-x}\left[\left(n+\eta_{1}+1\right) P_{n}^{\left(\eta_{1}, \eta_{2}\right)}(x)-(n+1) P_{n-1}^{\left(\eta_{1}, \eta_{2}\right)}(x)\right] \\
& P_{n}^{\left(\eta_{1}, \eta_{2}+1\right)}(x)=\frac{2}{2 n+\eta_{1}+\eta_{2}+2} \frac{1}{1+x}\left[\left(n+\eta_{2}+1\right) P_{n}^{\left(\eta_{1}, \eta_{2}\right)}(x)+(n+1) P_{n+1}^{\left(\eta_{1}, \eta_{2}\right)}(x)\right]
\end{aligned}
$$

Eq. (25) becomes

$$
\frac{d}{d x}\left|n>=\left(\frac{a_{1}-\kappa_{3}}{x}-\frac{\frac{1+A}{2}-\kappa_{2}}{1-x}\right)\right| n>+\left(\frac{\kappa_{1}}{x}+\frac{\kappa_{1}}{1-x}\right) \frac{A_{n}}{A_{n-1}} \mid n-1>,
$$

with

$$
\kappa_{1}=\frac{(n+A)\left(n+2 a_{1}\right)}{2 n+2 a_{1}+A} ; \kappa_{2}=\frac{n\left(n+2 a_{1}\right)}{2 n+2 a_{1}+A} ; \kappa_{3}=\frac{n(n+A)}{2 n+2 a_{1}+A},
$$

Rewriting Eq. (29) in the standard form

$$
\begin{array}{r}
{\left[x(1-x) \frac{d}{d x}+\left(a_{1}+\frac{1+A}{2}-\kappa_{2}-\kappa_{3}\right) x-a_{1}+\kappa_{3}\right]} \\
\times\left(\frac{2 n+2 a_{1}+A}{n+2 a_{1}}\right)\left|n>=(n+A) \frac{A_{n}}{A_{n-1}}\right| n-1>,
\end{array}
$$


we obtain the annihilation operator

$$
\hat{\Pi}_{-}=\left[x(1-x) \frac{d}{d x}+\frac{1}{2}\left(2 a_{1}+A-2\left(\kappa_{2}+\kappa_{3}\right)+1\right) x-a_{1}+\kappa_{3}\right]\left(\frac{2 n+2 a_{1}+A}{n+2 a_{1}}\right),
$$

with the eigenvalues

$$
\pi_{-}=(n+A) \frac{A_{n}}{A_{n-1}}
$$

which satisfy the eigenvalue equation in Eq. (24).

Following the same procedure and using the following relation [18]

$$
P_{n-1}^{\left(\eta_{1}, \eta_{2}\right)}(x)=P_{n}^{\left(\eta_{1}, \eta_{2}-1\right)}(x)-P_{n}^{\left(\eta_{1}-1, \eta_{2}\right)}(x),
$$

we immediately write the creation operator as

$$
\hat{\Pi}_{+}=\left[x(1-x) \frac{d}{d x}+\frac{1}{2}\left(2 a_{1}+A+2\left(\kappa_{4}-\kappa_{5}\right)+1\right) x-a_{1}-\kappa_{4}\right]\left(\frac{2 n+2 a_{1}+A+2}{n+2 a_{1}+A+1}\right)
$$

where

$$
\kappa_{4}=\frac{\left(n+2 a_{1}+A+1\right)\left(n+2 a_{1}+1\right)}{2 n+2 a_{1}+A+2} ; \kappa_{5}=\frac{\left(n+2 a_{1}+A+1\right)(n+A+1)}{2 n+2 a_{1}+A+2},
$$

with the eigenvalues

$$
\pi_{+}=(n+1) \frac{A_{n}}{A_{n+1}}
$$

Now, let us study the commutation relations of the operators $\hat{\Pi}_{\mp}$ to present the Lie algebra related with the two-term molecular potential. For this aim, we define the operator

$$
\hat{\Pi}_{0}=\hat{n}+\frac{1+A}{2}
$$

where $\hat{n}$ is the number operator satisfying

$$
\hat{n}|n>=n| n>
$$

So, the operator $\hat{\Pi}_{0}$ has the eigenvalues

$$
\pi_{0}=n+\frac{1+A}{2}
$$

By using Eqs. (32), (35), (38) and with the help of Eqs. (33), (37) and (40), we obtain the commutators as

$$
\left[\hat{\Pi}_{-}, \hat{\Pi}_{+}\right]=2 \hat{\Pi}_{0} ;\left[\hat{\Pi}_{0}, \hat{\Pi}_{+}\right]=\hat{\Pi}_{+} ;\left[\hat{\Pi}_{-}, \hat{\Pi}_{0}\right]=\hat{\Pi}_{-}
$$


which correspond to the Lie algebra associated with a $\mathrm{SU}(1,1)$ group.

Finally, we could write the Casimir operator of the group by using the above results as

$$
\hat{C}=-\hat{\Pi}_{-} \hat{\Pi}_{+}+\hat{\Pi}_{0}\left(\hat{\Pi}_{0}+1\right)=-\hat{\Pi}_{+} \hat{\Pi}_{-}+\hat{\Pi}_{0}\left(\hat{\Pi}_{0}-1\right),
$$

with the eigenvalue equation

$$
\hat{C}\left|n>=c_{0}\right| n>;
$$

where

$$
c_{0}=c(c-1) ; \quad c=\frac{1+A}{2} .
$$

\section{CONCLUSIONS}

We have computed the exact bound state energy eigenvalues and the corresponding normalized eigenfunctions of the two-term diatomic potential by using the NU method. Then we have obtained the step-up and step-down operators of the potential and shown that the ladder operators satisfy the Lie algebra of a $\mathrm{SU}(1,1)$ group. The Casimir operator of the group is also obtained and the eigenvalue equation is given.

\section{ACKNOWLEDGMENTS}

This research was partially supported by the Scientific and Technical Research Council of Turkey.

\section{Appendix A: Nikiforov Uvarov Method}

The Schrödinger equation can be transformed by using appropriate coordinate transformation into following form

$$
\sigma^{2}(y) \frac{d^{2} \Psi(y)}{d y^{2}}+\sigma(y) \tilde{\tau}(y) \frac{d \Psi(y)}{d y}+\tilde{\sigma}(y) \Psi(y)=0,
$$

where $\sigma(y)$ and $\tilde{\sigma}(y)$ are polynomials, at most, second degree, and $\tilde{\tau}(y)$ is a first-degree polynomial. By using the separation of variables, the solution is written as

$$
\Psi(y)=\psi(y) \varphi(y),
$$


which gives Eq. (A1) as a hypergeometric type equation [5]

$$
\frac{d^{2} \varphi(y)}{d y^{2}}+\frac{\tau(y)}{\sigma(y)} \frac{d \varphi(y)}{d y}+\frac{\lambda}{\sigma(y)} \varphi(y)=0,
$$

where $\psi(y)$ is defined by using the equation [5]

$$
\frac{1}{\psi(y)} \frac{d \psi(y)}{d y}=\frac{\pi(y)}{\sigma(y)}
$$

and the other part of the solution in Eq. (A2) is given by

$$
\varphi_{n}(y)=\frac{a_{n}}{\rho(y)} \frac{d^{n}}{d y^{n}}\left[\sigma^{n}(y) \rho(y)\right]
$$

where $a_{n}$ is a normalization constant and $\rho(y)$ is the weight function and satisfies the following equation [5]

$$
[\sigma(y) \rho(y)]^{\prime}=\tau(y) \rho(y)
$$

where prime denotes the derivative to coordinate.

The function $\pi(y)$ and the parameter $\lambda$ in the above equation are defined as

$$
\begin{aligned}
\pi(y) & =\frac{1}{2}\left[\sigma^{\prime}(y)-\tilde{\tau}(y)\right] \pm \sqrt{\frac{1}{4}\left[\sigma^{\prime}(y)-\tilde{\tau}(y)\right]^{2}-\tilde{\sigma}(y)+k \sigma(y)} \\
\lambda & =k+\pi^{\prime}(y)
\end{aligned}
$$

In the NU method, the square root in Eq. (A7) must be the square of a polynomial, so the parameter $k$ can be determined. Thus, a new eigenvalue equation becomes

$$
\lambda=\lambda_{n}=-n \tau^{\prime}(y)-\frac{1}{2} n(n-1) \sigma^{\prime \prime}(y) .
$$

where prime denotes the derivative and the derivative of the function $\tau(y)=\tilde{\tau}(y)+2 \pi(y)$ should be negative. 
[1] J. X. Sun, Acta Phys. Sin. 48, 1992 (1999).

[2] C. S. Jia, J. Y. Wang, S. He and L. T. Sun, J. Phys. A 33, 6993 (2000).

[3] F. Benamira, L. Guechi, S. Mameri and M. A. Sadoun, J. Math. Phys. 48, 032102 (2007).

[4] A. Arda and R. Sever, J. Math. Chem. DOI: 10.1007/s10910-012-0011-0 (in press).

[5] A. F. Nikiforov, and V. B. Uvarov, Special Functions of Mathematical Physics , (Birkhäuser, Basel, 1988).

[6] L. Infeld and T. E. Hull, Rev. Mod. Phys. 23, 21 (1951).

[7] Y. B. Ding, J. Phys. A 20, 6293 (1987).

[8] E. Schrödinger, Proc. R. Irish Acad. 47A, 53 (1941).

[9] S. H. Dong, App. Math. Lett. 16, 199 (2003).

[10] S. H. Dong, R. Lemus and A. Frank, Int. J. Quant. Chem. 86, 433 (2002).

[11] S. H. Dong and Z. Q. Ma, Am. J. Phys. 70, 520 (2002).

[12] S. H. Dong and Z. Q. Ma, Int. J. Mod. Phys. E 11, 155 (2002).

[13] S. H. Dong, G. H. Sun and M. Lozada-Cassou, Phys. Lett. A 328, 299 (2004).

[14] S. H. Dong, Can. J. Phys. 80, 129 (2002).

[15] S. H. Dong, G. H. Sun and Y. Tang, Int. J. Mod. Phys. E 12, 809 (2003).

[16] S. H. Dong, G. H. Sun and M. Lozada-Cassou, Int. J. Mod. Phys. AE 20, 5663 (2005).

[17] S. Flügge, Practical Quantum Mechnics I (Springer Verlag, Berlin, Heidelberg, New York, 1971).

[18] M. Abramowitz, I. A. Stegun, Handbook of Mathematical Functions with Formulas, Graphs, and Mathematical Tables (Dover Publications, New York, 1965) 